\title{
THE SPATIAL CONTROL OF GANGLIONIC NEURITE GROWTH BY THE SUBSTRATE-ASSOCIATED MATERIAL FROM CONDITIONED MEDIUM: AN EXPERIMENTAL MODEL OF HAPTOTAXIS ${ }^{1}$
}

\author{
FRANK COLLINS ${ }^{2}$ AND MARK R. LEE \\ Department of Anatomy, University of Utah School of Medicine, Salt Lake City, Utah 84132
}

Received January 17, 1984; Revised May 3, 1984; Accepted May 10, 1984

\begin{abstract}
Techniques are described for forming on the culture substrate a narrow pathway of material derived from medium conditioned by embryonic non-neuronal cells. There is a precise correlation between the known location of the pathway and the spatial distribution of neurites extended from whole ciliary ganglia or dissociated neurons grown on the patterned substrate. When individual growth cones moving on the pathway contact one of its edges, they turn sharply so as to remain on the pathway. This turning response is not simply due to a physical barrier to movement across the edge, since growth cones can cross onto the pathway when entering from the adjacent untreated substrate. The strong preference for neurites to maintain contact with the pathway also is not simply a result of their inability to survive or elongate outside of the pathway, since neurites from whole ganglia elongate readily on other regions of the substrate, although their growth is then undirected. Direct contact with the pathway is required for these directive effects, and there is no indication that the material bound to the substrate diffuses off and exerts its effects at a distance. The neurite-guiding activity is found in partially purified fractions of conditioned medium which also contain a substrate-bound inducer of neurite outgrowth. It is possible that both activities may be due to the same components of conditioned medium. These results suggest that ciliary ganglion growth cones are able to detect specific components of conditioned medium on the substrate and respond to their presence by changing their direction of movement so as to remain in contact with these components. This system should prove useful for channeling ganglionic outgrowth toward specific targets to establish desired patterns of innervation in vitro. It may also serve as a model system for studying the chemistry and mode of action of those components of the embryonic substrate which support and direct neurite outgrowth during development.
\end{abstract}

Heart cell conditioned medium is a powerful inducer of axon outgrowth from dissociated ciliary ganglion neurons in culture (Helfand et al., 1976). The induction of outgrowth requires the deposition of material from the conditioned medium onto the polyornithine-coated culture substrate (Collins, 1978b). This substrate-bound material produced by embryonic heart cells is sufficient to support axon outgrowth in the absence of any other components of conditioned medium (Collins 1978b, 1980). Based on its mode of action we have termed this material a substrate-conditioning factor (SCF). Recent studies have suggested that the activity of a similar SCF is associated with a class of proteoglycan typically found at the cell surface and in the extracellular matrix (Lander et al., 1982).

Although SCF is required for the initiation of neurite growth from dissociated neurons, it was unclear from these experiments whether continued elongation requires the continued presence of SCF. Since SCF binds to the substrate it cannot be

\footnotetext{
${ }^{1}$ We wish to thank Drs. Marcus Jacobson and Gary Schoenwolf for helpful comments on the manuscript and also Margaret Vogel for her capable technical assistance. This work was supported by grants from the National Institutes of Health and the National Science Foundation.

${ }^{2}$ To whom correspondence should be addressed.
}

easily removed in order to answer this question. This question could be approached, however, by observing what happens when neurites attempt to cross from an SCF-coated region of the substrate onto an uncoated region. To implement this approach experimentally, the border between the SCF-coated and uncoated regions of the substrate should be sharp (with respect to the dimensions of a growth cone) and the border's location should be known precisely, so that a firm correlation can be established between the behavior of the elongating axon and contact with the border. We have developed techniques for coating a narrow strip of the substrate with SCF which results in sharp borders whose location can be mapped precisely (Collins and Garrett, 1980; Collins, 1984a). The present report describes the active turning response of growth cones as they contact the edge of a substrate pathway of SCF. This prevents their movement off the pathway and results in the precise spatial control of ganglionic neurite growth by the pathway.

\section{Materials and Methods}

Culture conditions. Ciliary ganglia were removed from 8- to 9-day White Leghorn chicken embryos and dissociated into single cells by exposure to trypsin (Collins, 1978a). Dissociated ganglia were incubated in plastic tissue culture dishes (Falcon) for 3 to $4 \mathrm{hr}$ after dissociation to remove non-neuronal cells, which adhere to the dish, from neurons 
which do not adhere (Collins, 1980). Culture medium for dissociated neurons consisted of Ham's F12 (Grand Island Biological Co.) supplemented with $10 \%$ fetal calf serum (Irvine Scientific). For experiments involving intact ganglia the above medium was supplemented with $5 \%$ chicken embryo extract (Paul, 1965) to promote neuronal survival. Before use, medium containing the embryo extract was assayed (Collins and Dawson, 1982) to be certain that it contained no substrate-conditioning activity (which might interfere with the substrate pathway). Cultures of intact ganglia were incubated at $37^{\circ} \mathrm{C}$ in a humidified atmosphere with $\mathrm{CO}_{2}$ levels adjusted for a pH between 7.2 and 7.4. When cultures of dissociated neurons were incubated on the microscope stage, the dish lid was sealed with silicone vacuum grease to retard evaporation and $\mathrm{pH}$ change. The temperature was maintained at $37^{\circ} \mathrm{C}$ with an air curtain incubator.

Partial purification of SCF. Confluent monolayers of trypsin-dissociated heart cells from 9- to 10-day chicken embryos were exposed to serum-free Ham's F12 for 48 to 72 hr (Collins, 1978a). After centrifugation at $15,000 \times g$ for $45 \mathrm{~min}$ to remove any cellular debris, the inducer of neurite outgrowth (Collins, 1978b) was partially purified as follows (see Table I): $250 \mathrm{ml}$ of serum-free heart conditioned medium were concentrated and freed of small molecules by retention on an Amicon filtration membrane with a nominal 100,000-dalton cutoff. The concentrated material was then placed on a $1.5 \times 80 \mathrm{~cm}$ column of Sepharose CL-2B (Pharmacia Fine Chemicals) and eluted with $0.1 \mathrm{M}$ $\mathrm{NaCl}, 0.05 \mathrm{M}$ Tris, $\mathrm{pH}$ 7.0. A single peak of biological activity emerged after the void volume. The peak fraction was applied to a 2 -ml column of DEAE-Sepharose (Pharmacia) and unbound materials washed through with the Sepharose CL-2B elution buffer. All biological activity remained bound to the column. A discontinuous gradient of increasing $\mathrm{KCl}$ from 0.2 to $1.0 \mathrm{M}$ in $0.2 \mathrm{M}$ steps was applied to the column. The $0.6 \mathrm{M} \mathrm{KCl}$ fraction contained more than $85 \%$ of the biological activity recovered from the DEAE column; the remainder of the activity was found in the $0.4 \mathrm{M} \mathrm{KCl}$ fraction. Before testing, the various $\mathrm{KCl}$ fractions were passed over a Sephadex G-25 column (Pharmacia) equilibrated with the CL-2B buffer in order to remove excess salt. The $0.6 \mathrm{M} \mathrm{KCl}$ fraction was used in the experiments reported here diluted 1:25 with Hanks' balanced salt solution. Table I gives yields and specific activities at various stages of purification and Figure 6 shows the electrophoretic profiles of the starting material, the $0.6 \mathrm{M}$, and the inactive $0.2 \mathrm{M} \mathrm{KCl}$ fractions on sodium dodecyl sulfate (SDS)-polyacrylamide gels. Assays of biological activity were performed as described in the legend to Table I (see also Collins and Dawson, 1982). Protein deteminations were done using the method of Bradford (1976).

Formation of a pathway o substrate-bound material. Plastic tissue culture dishes ( $35 \mathrm{~mm}$; Falcon) were coated with polyornithine as previously described (Collins, 1978a). "Wicks" were made in one of two ways. (1) A glass capillary tube was heated and, while still soft, was drawn into a thin hair. A length of uniform diameter was separated from the rest and sealed at both ends by rapid insertion in a flame. (2) A narrow, rectangular strip was cut from a piece of Whatman No. 1 filter paper as previously described (Collins and Garrett, 1980). Glass hairs allowed the formation of narrower pathways with straighter borders. The dish was supported at an angle approximately $45^{\circ}$ to the horizontal, and a wick was laid onto the dry surface of the polyornithine-coated dish. The fraction whose activity was being tested $(0.1$ $\mathrm{ml}$ ) was placed in contact with the lower end of the wick. The liquid

\section{TABLE I}

\section{Partial purification of SCF}

Serum-free heart cell conditioned medium was fractionated as described under "Materials and Methods." Biological activity was determined by treating polyornithine-coated culture wells overnight with serial 2 -fold dilutions of each fraction. Dishes were washed to remove unbound materials and dissociated ciliary ganglion neurons plated on in unconditioned medium. A biological unit (U) is defined as the reciprocal of the highest dilution that still gave greater than $10 \%$ initiated neurons.

\begin{tabular}{lcccc}
\hline & $\begin{array}{c}\text { Total } \\
\text { Volume }\end{array}$ & $\begin{array}{c}\text { Biological } \\
\text { Activity }\end{array}$ & $\begin{array}{c}\text { Protein } \\
\text { Content }\end{array}$ & $\begin{array}{c}\text { Specific } \\
\text { Activity }\end{array}$ \\
\hline & $m l$ & $U / m l$ & $\mu g / m l$ & \\
Starting material & 250 & 32 & 168 & $5.25 \mu \mathrm{g} / \mathrm{U}$ \\
Separose CL-2B peak & 12 & 512 & 113 & $221 \mathrm{ng} / \mathrm{U}$ \\
$0.6 \mathrm{M}$ KCl DEAE fraction & 0.5 & 4096 & 184 & $45 \mathrm{ng} / \mathrm{U}$ \\
\hline
\end{tabular}

was drawn up along the wick by capillary action and extended as a straight liquid border to either side of the edge of the glass hair or filter paper. A lid was then sealed to the dish with silicone vacuum grease to prevent evaporation of the solution, and the whole was placed overnight at $4^{\circ} \mathrm{C}$. After approximately $16 \mathrm{hr}$, the dish was placed on the stage of an inverted microscope and the location of the pathway and its borders was recorded as described in the legends to Figures 1 to 3 . The dish was then flooded with unconditioned culture medium, the now floating glass hair was removed, and the dish was washed a total of three times with fresh unconditioned medium to remove unbound components and leave a pathway of substrate-bound material, the location of whose borders corresponds to the location of the edges of the original liquid strip.

Iodination. Iodination was carried out by mixing in order $10 \mu \mathrm{l}$ of sample, $2 \mu$ l of $0.1 \mathrm{M}$ phosphate buffer $(\mathrm{pH} 7.5), 1.2 \mathrm{mCi}$ of $\mathrm{Na}^{125} \mathrm{I}$ in 2 $\mu \mathrm{l}$, and $7.5 \mu \mathrm{l}$ of chloramine $\mathrm{T}(300 \mu \mathrm{g} / \mathrm{ml}$ in phosphate buffer) for 45 sec on ice. The reaction was stopped by addition of $7.5 \mu \mathrm{l}$ of sodium metabisulfite $(600 \mu \mathrm{g} / \mathrm{ml}$ in phosphate buffer $)$. The reaction mixture was diluted to approximately $0.2 \mathrm{ml}$ and placed onto a $3-\mathrm{ml}$ column of Sephadex G-25 which had been previously exposed to $1 \mathrm{ml}$ of fetal calf serum to reduce nonspecific adsorbtion. The column was eluted with phosphate buffer, and the first peak of radioactivity was collected $(0.3$ $\mathrm{ml}$ ). Greater than $94 \%$ of the radioactivity in this fraction was precipitated in $10 \%$ trichloroacetic acid using bovine serum albumin as carrier.

Polyacrylamide gel electrophoresis. Slab gels, containing a 10 to $25 \%$ linear gradient of polyacrylamide, were prepared according to the method of Studier (1973) and were used with the discontinuous buffer system of Laemmli (1970) containing $0.1 \%$ SDS. A $5 \%$ polyacrylamide spacer gel was used on top of the separating gel. Samples were prepared in buffer containing $2 \%$ SDS and $2 \% 2$-mercaptoethanol and heated to $95^{\circ} \mathrm{C}$ for $2 \mathrm{~min}$. Gels were fixed, then vacuum dried and used to expose $\mathrm{x}$-ray film for autoradiography.

\section{Results}

Confinement of neurites within the pathway. Intact ciliary ganglia were placed on a pathway of SCF deposited either from unfractionated heart conditioned medium (Fig. 1) or from a partially purified fraction of the conditioned medium (Fig. 2). Those neurites which emerged from the ganglion and made contact with the SCF pathway remained confined within its borders and continued to elongate away from the ganglion. Consequently, the advancing neurites gradually filled the pathway further and further away from the ganglion (Fig. 1). Such pathways could conceivably be used to connect ganglia with desired targets for innervation which, in the case of Figure 2, is represented by a second ciliary ganglion. It should be kept in mind that in these and all subsequent experiments the location of the substrate pathway was known independently of the behavior of the neurites and was confirmed by that behavior (Figs. 1 and 3).

Axons that did not make contact with the pathway, because they emerged from regions of the ganglion's perimeter not in contact with the SCF, continued to elongate but showed no signs of channeled outgrowth, indicating that direct contact with the pathway is necessary for this response. The observation that the untreated polyornithine substrate supports ganglionic neurite growth (Collins, 1984b) also indicates that the confined growth of those neurites which enter the pathway is not simply due to their inability to elongate outside the pathway.

At the time the photographs shown in Figures 1 and 2 were taken, thick neurite cables had formed along the edges of the pathway near the ganglia. Only at this time were neurites observed to leave the pathway in significant numbers. The dense network of neurites may have simply prevented additional neurites from coming into direct contact with the pathway. Before the density of neurites became so great, neurites were as well confined within the pathway near the ganglion as they still were at later times more distally (Fig. 1).

One additional feature of the results illustrated in Figure 1 


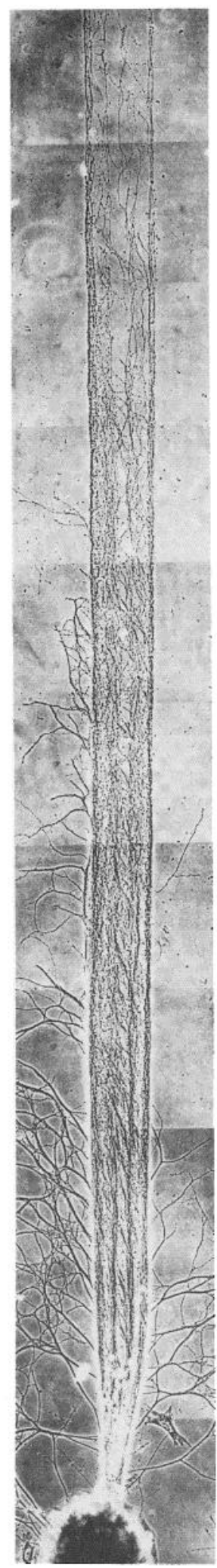

Figure 1. The channeling of ganglionic neurite growth along a substrate pathway of conditioned medium. A pathway of treated substrate was prepared using a glass hair and heart cell conditioned medium. The dish was fastened in a calibrated holder on the stage of an inverted microscope, and the location of both edges of the liquid strip, at intervals along the length of the strip, was determined by aligning each liquid edge (see bottom frame in Fig. 3) with a line in an eyepiece reticle and recording the corresponding reading on special vernier stage micrometer scales (Collins and Garrett, 1980). At any time after the dish

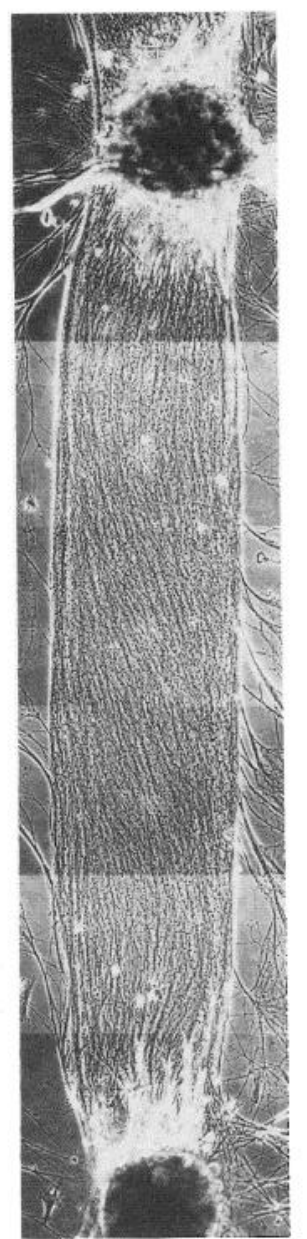

Figure 2. Channeling of ganglionic neurite growth by a partially purified fraction of conditioned medium. A pathway of treated substrate was formed using a filter paper wick and partially purified SCF (the $0.6 \mathrm{M} \mathrm{KCl} \mathrm{DEAE}$ fraction of conditioned medium). The location of the pathway was recorded as in Figure 1. Two ciliary ganglia are shown in this photograph taken $94 \mathrm{hr}$ after plating. Magnification $\times 70$.

requires comment. The tendency of neurites to remain near the edges and away from the center of the pathway is probably an artifact of the method used to create the pathway in this particular experiment. Use of the glass hair in forming the border (see "Materials and Methods") tends to exclude the SCF solution from the center of the forming pathway and thus causes more SCF to be deposited near the edges than centrally. When the pathway is formed using the filter paper strip (see "Materials and Methods"), the distribution of neurites within the pathway is more uniform (Fig. 2).

Growth cones turn at the borders. One possible explanation for the confinment of neurite outgrowth to the pathway in the previous experiments is that the growth cones of elongating neurites turn at the edge of the pathway so as to remain in contact with the SCF. To determine whether this was happening, dissociated ciliary ganglion neurons were plated onto a region of the pathway whose border had been photographed

was washed and ciliary ganglia were plated on in unconditioned medium, the dish could be fastened in the original position in its holder and the stage micrometer scales and eyepiece reticle line used to relocate the region of substrate which had been exposed to conditioned medium. In this way the location of the pathway of SCF could be compared to the spatial distribution of neurites. A portion of one ganglion is shown at the bottom of the figure, which was taken $72 \mathrm{hr}$ after plating. Magnification $\times 48$. 


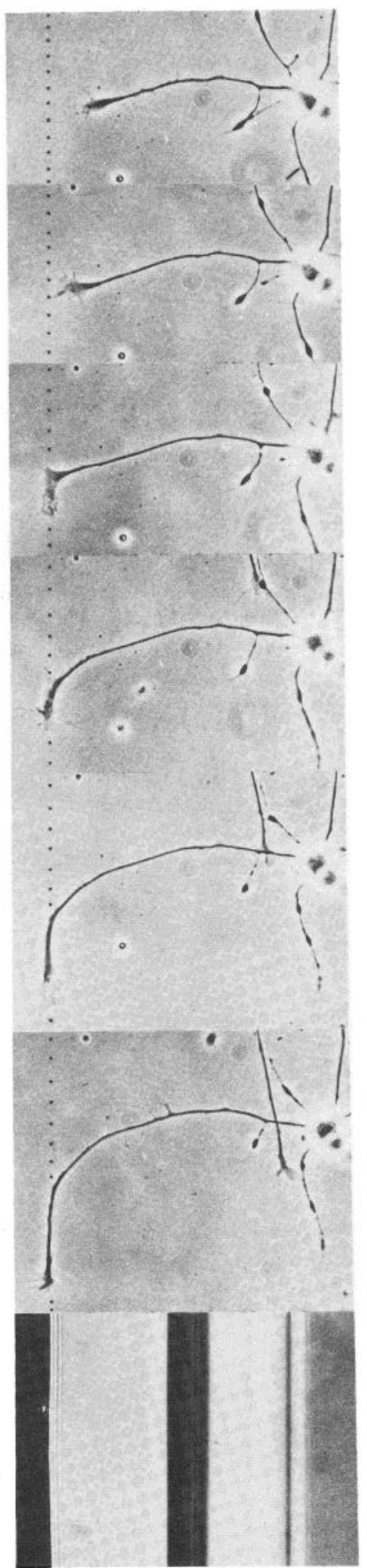

Figure 3. The turning response of growth cones upon contact with the edgeof the SCF pathway. A pathway of treated substrate was formed using a glass hair and conditioned medium. The dish was fastened in a holder on the stage of an inverted microscope. A segment of the liquid strip including one edge was located in the microscope field and photographed (bottom frame). In this frame the edge of the liquid strip is the sharp line separating the region of white (liquid) at the right from the black region (untreated substrate) at the left. The dark shadows in the white region are optical reflections of the glass hair. The dish was washed and dissociated ciliary ganglion neurons were previously (bottom frame, Fig. 3). Neurons which landed on the pathway (i.e., to the right of the dotted line in Fig. 3) extended neurites whose growth cones were observed as they approached the known edge of the pathway. The dotted line in Figure 3 indicates for convenient reference the approximate position of the border in each frame rather than a tracing of its exact position, which varies slightly across the field, as can be seen in the bottom frame.

Before directly contacting the border, growth cones showed no tendency to change direction sharply (frames 1 and 2, Fig. 3 ). However, when the leading edge of the growth cone contacted the border, the growth cone showed obvious signs of turning (frame 3, Fig. 3). Growth cones then continued to elongate on the pathway adjacent to the border (frames 4 to 6 , Fig. 3), often subsequently leaving the edge to elongate more centrally on the pathway. The growth cones we have observed turned at the border in a direction which caused the neurite to bend the least.

Directed initiation from neurons straddling the border. Nerve cell bodies occasionally attached to the substrate just at the location of the border so that a part of the cell body lay in direct contact with the pathway, while the rest of the cell lay on the untreated substrate. In such nerve cells (three observations), neurites emerged only from that region of the soma in direct contact with the pathway (Fig. 4).

Controls for the growth cone turning response. It is important to determine whether neurite outgrowth is channeled, in the experiments described above, simply because the borders of the pathway are inadvertently created mechanical barriers which prevent neurites from crossing. We addressed this potential artifact in our previous preliminary description of the channeling of neurite outgrowth from dissociated neurons along a substrate pathway of conditioned medium (Collins and Garrett, 1980). In those experiments, pathways were formed using a filter paper strip (see "Materials and Methods"), but neurons were subsequently cultured on them in heart conditioned medium rather than unconditioned medium (Collins and Garrett, 1980). The deposition of material from the conditioned medium on all parts of the substrate would have eliminated the chemical distinction between the pathway and the rest of the substrate but should not have been more successful than regular medium at eliminating a physical barrier to outgrowth. Nevertheless, the neurites crossed freely over the location of the borders when cultured in conditioned medium, but not in unconditioned medium, suggesting that there was no physical barrier (Collins and Garret, 1980).

In the present work we performed additional experiments which indicate that there is no physical barrier to neurite movement at the borders. Dishes in which a pathway of SCF had been formed as usual were subsequently treated with a solution containing a low level of SCF activity (Fig. 5). The small amount of SCF deposited from this solution onto the substrate outside the original pathway allowed some of the neurons to initiate outgrowth off the pathway. Without this pretreatment of the whole dish, such outgrowth off the pathway failed to occur, even immediately adjacent to the pathway. This procedure made it possible in several instances to follow individual neurites which had approached the edge of the pathway from the untreated substrate. In these instances (five observa-

plated on in unconditioned medium while the dish remained fastened in place. Since the dish had not been moved, the location of the edge of treated substrate was unchanged from its position when photographed. The dotted line indicates the location of that edge within each field; the region of SCF-treated substrate is to the right of this line. The upper frames (frames 1 to 6 ) were taken at intervals after the addition of neurons. The duration from the topmost frame to the next to the bottom was $62 \mathrm{~min}$. Magnification $\times 360$. 

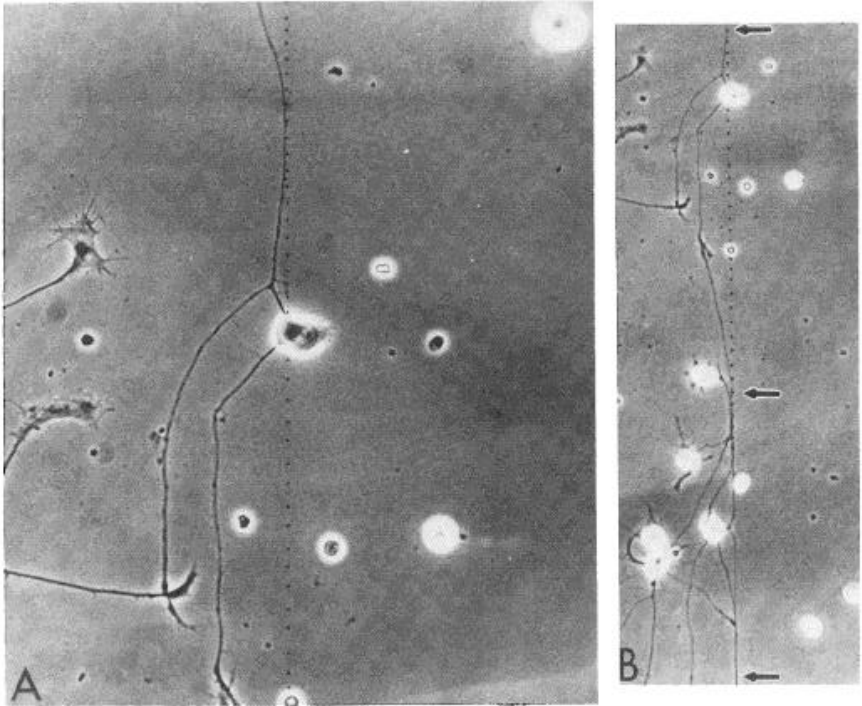

Figure 4. The effect of localized contact of the neuronal soma with SCF. A pathway of treated substrate was prepared using a glass hair and partially purified SCF (the $0.6 \mathrm{M} \mathrm{KCl}$ DEAE fraction). The edge of the pathway was recorded as in Figure 3 and is indicated by the dotted lines in $A$ and $B$. The pathway of treated substrate extends to the left of the dotted line. The dish was washed and dissociated ciliary ganglion nerve cells were plated on in unconditioned medium. The neuronal soma in the center of $A$ attached so that one portion of the cell was in contact with the SCF pathway to the left of the dotted line, while the rest of the cell lay in contact with the untreated substrate to the right. Neurites emerged only from that portion of the soma in direct contact with the SCF. $B$ shows a view of the same region at lower power, including the cell straddling the border in $A$, now near the top in $B$. It can be seen that the previously determined location of the edge of the SCF pathway (dotted line) is confirmed independently by the behavior of the neurites, which are deflected at this border (arrows in $B)$. Magnifications: $A, \times 450 ; B, \times 220$.

tions), the neurites crossed freely over the border onto the pathway (Fig. 5). The instance illustrated in Figure 5 is particularly fortunate, since the neurite which crossed onto the pathway subsequently branched, and several of these branches later approached the edge of the pathway, this time from the "inside" (Fig. 5, arrows). These neurite branches were deflected as usual from crossing off the pathway, indicating that the initial crossing of this neurite onto the pathway was not due to some peculiarity of its makeup that rendered it incapable of responding to the SCF pathway. Thus, growth cones can apparently cross the borders of the pathway when going from a lower to a higher concentration of the substrate-bound materials from conditioned medium, but movement in the opposite direction is much less likely due to the growth cone turning response previously described. There is no purely physical barrier to neurite crossing.

The chemical specificity of haptotaxis. The substrate-bound inducer of neurite outgrowth, which we have termed SCF, and the agent promoting haptotaxic neurite guidance were found in the same fractions of heart conditioned medium during the process of partial purification of the former activity (as described under "Materials and Methods" and in Table I). Almost all of the detectable neurite-inducing SCF activity was found in the $0.6 \mathrm{M} \mathrm{KCl} \mathrm{DEAE} \mathrm{fraction} \mathrm{(Fig.} \mathrm{6,} \mathrm{lane} C$ ), with a small amount of activity, not greater than $15 \%$ of the total, occasionally present in the $0.4 \mathrm{M} \mathrm{KCl}$ fraction (not shown). These fractions were the only ones which affected the spatial distribution of neurites when used to form a substrate pathway (Figs. 2,4 , and 5). Fractions of conditioned medium, such as the 0.2 $\mathrm{M} \mathrm{KCl} \mathrm{DEAE} \mathrm{fraction} \mathrm{(Fig.} \mathrm{6,} \mathrm{lane} \mathrm{B)} \mathrm{that} \mathrm{lacked} \mathrm{detectable}$

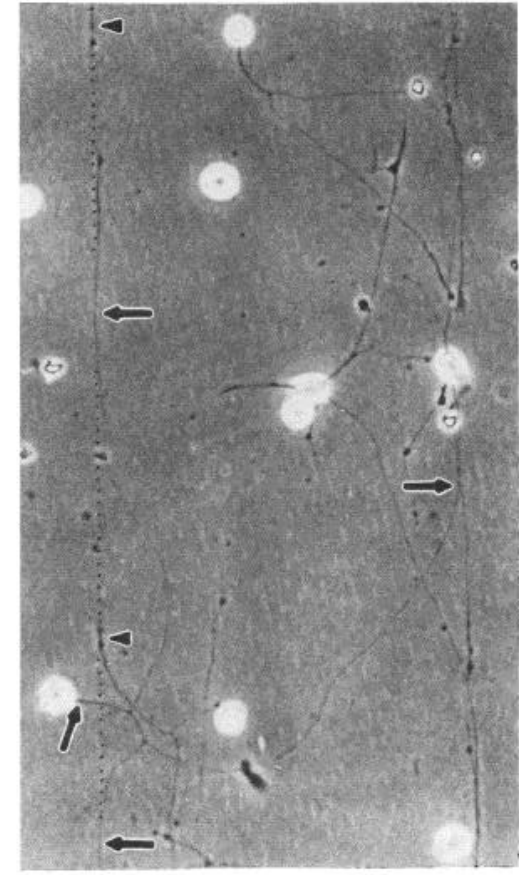

Figure 5. The behavior of neurites approaching the border from the untreated substrate. A pathway of treated substrate was prepared and the location of one edge was recorded as in Figure 4. The left edge of the pathway is indicated by the dotted line, while the right edge corresponds to the position of the deflected neurites. The dish was washed with Hanks' balanced salt solution to remove the glass hair and unbound components of the $0.6 \mathrm{M} \mathrm{KCl} \mathrm{DEAE} \mathrm{fraction.} \mathrm{The} \mathrm{entire}$ substrate was then treated for $2 \mathrm{hr}$ with a solution of the $0.6 \mathrm{M} \mathrm{KCl}$ fraction diluted 1:100 with balanced salt solution. The dishes were again washed and dissociated neurons were plated on in unconditioned medium. The nerve cell at the lower left attached outside the original SCF pathway but nevertheless extended a neurite because of the subsequent treatment of the entire substrate with low levels of SCF. The arrows indicate the course followed by this neurite as it crossed freely from the untreated substrate onto the SCF pathway, branched on the pathway, and those branches were deflected as they recontacted the same border from "inside" the pathway. The arrowheads indicate other neurites whose deflection at the border also confirms its location. Magnification $\times 170$.

neurite-inducing activity, also failed to affect the distribution of ganglionic neurite outgrowth when used to form a substrate pathway.

Comparison of lane $C$ in Figure 6 with the starting material in lane $A$ indicates that a substantial number of iodinatable components present in the latter have been greatly reduced in relative amount during the procedures used to produce the 0.6 $\mathrm{M} \mathrm{KCl}$ fraction. The extent of purification is also indicated by the results in Table I. The modest increase in specific activity during partial purification (about 100-fold; Table I) indicates that the $0.6 \mathrm{M} \mathrm{KCl}$ fraction is probably still rather impure, and there is no evidence linking any of the hands visible in this fraction (Fig. 6, lane $C$ ) with either of the biological activities discussed above. Nevertheless, the results are consistent with the possibility that neurite guidance and the induction of neurite growth are due to specific, and perhaps the same, components of conditioned medium.

\section{Discussion}

Our results indicate that there is a difference between neurite initiation and neurite elongation with respect to the requirement for SCF. Neurite initiation from dissociated neurons requires $\mathrm{SCF}$, as indicated by the absence of growth cone 


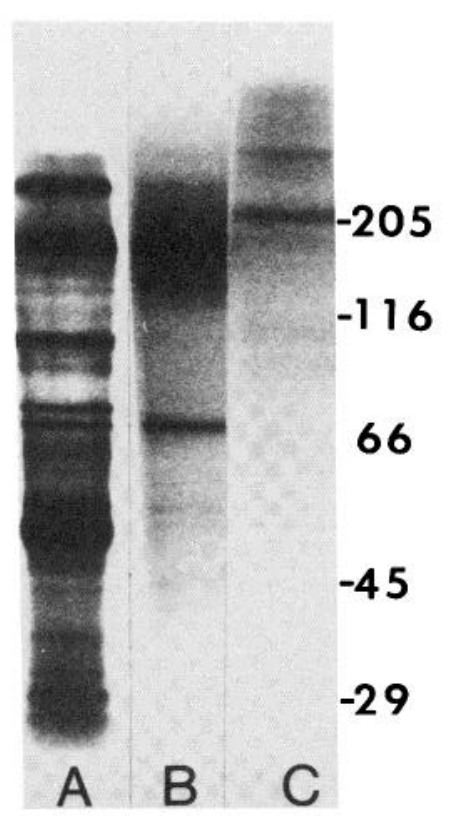

Figure 6. Electrophoretic profiles of fractions of heart conditioned medium during partial purification. Serum-free heart conditioned medium was partially purified as described under "Materials and Methods" and in Table I. Equal aliquots of conditioned medium concentrated by Amicon filtration (lane A), the $0.2 \mathrm{M} \mathrm{KCl}$ (lane B), and the $0.6 \mathrm{M}$ $\mathrm{KCl}$ (lane $C$ ) DEAE eluate fractions were iodinated and run on SDSpolyacrylamide gradient slab gels under reducing conditions, and the dried gels were used to prepare the autoradiograms illustrated here. Lanes $B$ and $C$ were exposed for equal times (1 week), but lane $A$ was given an exposure of only $12 \mathrm{hr}$ to maintain visibility of the bands. The position of molecular weight standards in kilodaltons is indicated to the right of lane $C$.

formation on untreated polyornithine substrates (Collins, 1978a, b, 1980; Collins and Garrett, 1980). However, once initiation has occurred, neurites from dissociated neurons do not require SCF for continued elongation, as indicated by the ability of neurites which have crossed off of a pathway of this material to continue elongating far from the pathway (Collins and Garrett, 1980). Undissociated ganglia do not require SCF, either for neurite initiation or for continued elongation of neurites, both of which occur on untreated polyornithine substrates (Collins, 1984b). There is some evidence that ganglia can condition the substrate in their immediate vicinity (Adler and Varon, 1981); however, if this were happening to any considerable extent in the present experiments, it would have obliterated the previously formed substrate pathways.

The simplest assumptions to explain these results are the following. (1) Neurite initiation has special requirements not met by a polyornithine substrate in standard culture medium. (2) Substrate-bound materials from conditioned medium, or analogous materials within undissociated ganglia, supply the requirements for initiation. (3) Once initiation has occurred, neurites from dissociated neurons or intact ganglia can use polyornithine in standard culture medium as a suitable substrate for continued elongation.

Although SCF is not required for continued elongation, it does have an effect on neurite growth since there is a marked tendency for the spatial distribution of neurites to correspond to the spatial distribution of this substrate-bound material. As indicated above, the spatial correspondence is not due to an inability of neurites to elongate in the absence of the substratebound material. It is also not due to a physical barrier to neurite crossing at the borders of the pathway of SCF (Fig. 5; Collins and Garrett, 1980). Rather, the patterned distribution of neurites on a patterned substrate appears to be due to the "preference" of growth cones for contact with substrate-bound conditioned medium. Growth cones turn at the borders of the conditioned medium pathway so as to remain in contact with the substrate-bound material (Fig. 3).

The spatial correspondence between the distribution of neurites and the distribution of SCF indicates that the SCF remains bound to the substrate during the culture period in the same locations in which it was originally deposited from the liquid conditioned medium. The absence of relocation of the substrate-bound material is also indicated by the observation that the initiation of neurites from dissociated neurons does not occur off the pathway, even immediately adjacent to the SCF-treated substrate (Collins, 1978b; Collins and Garrett, 1980).

The ability of neurites to choose between alternative permissive substrates as described here is reminiscent of the ability of fibroblasts and neurites to associate selectively with the more adhesive of two artificial substrates of differing adhesivity (Carter, 1965; Harris, 1973; Letourneau, 1975). The interest of the present result lies in the fact that the material promoting directed outgrowth is derived from embryonic non-neuronal cells and is present in substantial quantities in medium conditioned by such cells (Collins, 1978b). Whether this material acts by increasing substrate adhesivity remains to be determined. The preliminary characterization of this material reported here indicates that the agent directing outgrowth and the agent promoting neurite initiation from dissociated neurons are both present in the same partially purified preparation of serum-free conditioned medium. It may thus be possible to eventually purify and characterize a cell product capable of initiating and directing neurite growth.

It may be worthwhile to clarify the relationship of the SCF described here to other components of heart cell conditioned medium that also affect ciliary ganglion neurons in culture. Thus, conditioned medium is known to prolong the survival of ciliary ganglion neurons (Helfand et al., 1976; Adler and Varon, 1980) and to increase their rate of neurite elongation (Collins and Dawson, 1982). The components of conditioned medium responsible for these effects are distinct from those described in the present report. The survival- and elongation-promoting agents act without attaching to the culture substrate and are readily separated by simple biochemical procedures from the SCF discussed here (Collins, 1978b; Adler and Varon 1980; Collins and Dawson, 1982).

Relationship to axonal guidance in vivo. Axonal guidance during embryogenesis probably results from the interplay of a number of different mechanisms (Katz and Lasek, 1980). There is direct evidence that one such mechanism is the laying down of spatially distinct pathways, composed either of non-neuronal cells or other axons, that growth cones are programmed to follow with a high degree of specificity (Raper et al., 1983). In these instances it appears that direct contact between the pathway and the responding growth cone is required for guidance by such pathways (Raper et al., 1983). Guidance presumably is mediated by specific surface components of the cells in contact. This mechanism of guidance by substrate-bound cues has been termed haptotaxis (Katz and Lasek, 1980). Since neurites in vivo can be made to follow abnormal pathways (e.g., Giorgi and Van der Loos, 1978; Katz and Lasek, 1981), the substrate preferences of growth cones apparently are not absolute. It seems instead that for each type of neuron there may by a heirarchical order of substrate preference, so that the distribution of neurites is determined by choices made between the substrates available at any one time.

The present culture system appears to be a useful in vitro 
model of axonal guidance by haptotaxis. We have demonstrated that the spatial distribution of neuritic outgrowth from a peripheral ganglion in vitro can be controlled by the spatial distribution of substrate-bound components from conditioned medium. The material remains bound to the substrate during the period of growth, and direct contact between the growth cone and the bound material is necessary for neuritic guidance. The aspect of heirarchical choice is also present since elongating neurites grow on the less preferred substrate (untreated polyornithine) when the substrate-bound conditioned medium is unavailable. The culture system we have described may thus provide a means to study the nature of the interactions between neuron and substrate that mediate axonal guidance as well as to determine the chemical characteristics and locations in vivo of the active components.

\section{References}

Adler, R., and S. Varon (1980) Cholinergic neuronotrophic factors. V. Segregation of survival and neurite promoting activities in heartconditioned medium. Brain Res. 188: 437-448.

Adler, R., and S. Varon (1981) Neurite guidance by polyornithineattached materials of ganglionic origin. Dev. Biol. 81: 1-11.

Bradford, M. M. (1976) A rapid and sensitive method for the quantitation of microgram quantities of protein utilizing the principle of protein-dye binding. Anal. Biochem. 72: 248-254.

Carter, S. B. (1965) Principles of cell motility and the directionality of cell movement and cancer invasion. Nature 208: 1183-1185.

Collins, F. (1978a) Axon initiation by ciliary neurons in culture. Dev. Biol. 65: 50-57.

Collins, F. (1978b) Induction of neurite outgrowth by a conditioned medium factor bound to the culture substratum. Proc. Natl. Acad. Sci. U. S. A. 75: 5210-5213.

Collins, F. (1980) Neurite outgrowth induced by the substrate associated material from nonneuronal cells. Dev. Biol. 79: 247-252.

Collins, F. (1984a) Multiple sites for the regulation of neurite out- growth. In Cellular and Molecular Biology of Neuronal Development, I. Black, ed., pp. 217-230, Plenum Press, New York.

Collins, F. (1984b) An effect of nerve growth factor on the parasympathetic ciliary ganglion. J. Neurosci. 4: 1281-1288.

Collins, F., and A. Dawson (1982) Conditioned medium increases the rate of neurite elongation: Separation of this activity from the substratum-bound inducer of neurite growth. J. Neurosci. 2: 10051010.

Collins, F., and J. E. Garrett, Jr. (1980) Elongating neurites follow a pathway of material released from embryonic nonneuronal cells. Proc. Natl. Acad. Sci. U. S. A. 77: 6226-6228.

Giorgi, P. P., and H. Van der Loos (1978) Axons from eyes grafted in Xenopus can grow into the spinal cord and reach the optic tectum. Nature 275: 746-748.

Harris, A. (1973) Behavior of cultured cells on substrata of variable adhesiveness. Exp. Cell Res. 77: 285-291.

Helfand, S. L., G. A. Smith, and N. K. Wessells (1976) Survival and development in culture of dissociated parasympathetic ncurons from ciliary ganglia. Dev. Biol. 50: 541-547.

Katz, M. J., and R. J. Lasek (1980) Guidance cue patterns and cell migration in multicellular organisms. Cell Motil. 1: 141-157.

Katz, M. J., and R. J. Lasek (1981) Substrate pathways demonstrated by transplanted Mauthner axons. J. Comp. Neurol. 195: 627-641.

Laemmli, U. K. (1970) Cleavage of structural proteins during the assembly of the head of bacteriophage T4. Nature 227: 680-685.

Lander, A. D., D. K. Fujii, D. Gospodarowicz, and L. F. Reichardt (1982) Characterization of a factor that promotes neurite outgrowth: Evidence linking activity to a heparan sulfate proteroglycan. J. Cell Biol. 94: 574-585.

Letourneau, P. C. (1975) Cell-to-substratum adhesion and guidance of axonal elongation. Dev. Biol. 44: 92-101.

Paul, J. (1965) Cell and Tissue Culture, p. 71, Williams and Wilkins, Baltimore.

Raper, J. A., M. Bastiani, and C. S. Goodman (1983) Pathfinding by neronal growth cones in grasshopper embryos. II. Selective fasciculation onto specific axonal pathways. J. Neurosci. 3: 31-41.

Studier, F. W. (1973) Analysis of bacteriophage T7 early RNAs and proteins on slab gels. J. Mol. Biol. 79: 237-248. 